\title{
Investigation on the processing and improving the cleavage efficiency of furin cleavage sites in Pichia pastoris
}

Yide Huang ${ }^{1 *} \mathbb{D}$, Yanyu Long ${ }^{1}$, Suhuan Li ${ }^{1}$, Ting Lin ${ }^{1}$, Jingwen Wu' ${ }^{1}$, Yafei Zhang ${ }^{1}$ and Yao Lin ${ }^{1} 2^{*}$

\begin{abstract}
Background: Proprotein convertase furin is responsible for the processing of a wide variety of precursors consisted of signal peptide, propeptide and mature peptide in mammal. Many precursors processed by furin have important physiological functions and can be recombinantly expressed in Pichia pastoris expression system for research, pharmaceutical and vaccine applications. However, it is not clear whether the furin cleavage sites between the propeptide and mature peptide can be properly processed in P. pastoris, bringing uncertainty for proper expression of the coding DNA sequences of furin precursors containing the propeptides and mature peptides.

Results: In this study, we evaluated the ability of P. pastoris to process furin cleavage sites and how to improve the cleavage efficiencies of furin cleavage sites in $P$. pastoris. The results showed that $P$. pastoris can process furin cleavage sites but the cleavage efficiencies are not high. Arg residue at position P1 or P4 in furin cleavage sites significantly affect cleavage efficiency in P. pastoris. Kex2 protease, but not YPS1, in P. pastoris is responsible for processing furin cleavage sites. Heterologous expression of furin or overexpression of Kex2 in P. pastoris effectively increased cleavage efficiencies of furin cleavage sites.
\end{abstract}

Conclusions: Our investigation on the processing of furin cleavage sites provides important information for recombinant expression of furin precursors in P. pastoris. Furin or Kex2 overexpressing strains may be good choices for expressing precursors processed by furin in P. pastoris.

Keywords: Furin, Pichia pastoris, Furin cleavage site, Proprotein convertase, Kex2, YPS1

\section{Background}

Many important peptides or proteins are synthesized intracellularly as higher-molecular-mass inactive precursors consisted of signal peptide, propeptide and mature peptide in eukaryotes from yeasts to mammals $[1,2]$. Release of propeptide from inactive precursor proteins are proteolyzed by proprotein convertases within the secretory pathway, leading to the production of biologically active proteins or peptides. The product of the KEX2 gene, Kex2 [also known as kexin (EC 3.4.21.61)], was the first identified proprotein convertase involved in

\footnotetext{
*Correspondence: ydhuang@fjnu.edu.cn; yaolin@fjnu.edu.cn

1 Provincial University Key Laboratory of Cellular Stress Response and Metabolic Regulation, College of Life Sciences, Fujian Normal University, Fuzhou 350007, China

Full list of author information is available at the end of the article
}

the processing of $\alpha$-mating factor and killer toxin precursors in Saccharomyces cerevisiae. Kex2 belonging to the subtilisin family has a catalytic domain similar to bacterial serine proteases and cleaves its precursors at paired basic residues [3]. Proprotein convertase furin, encoded by the fur gene, is the homologue of Kex2 in mammal [4]. Furin is expressed as a 794 amino acid precursor and rapidly converted into active mature form by two intramolecular autocatalytic cleavage (the first cleavage site, Arg-Ala-Lys-Arg ${ }^{107}$; the second cleavage site, Arg-Gly-Val-Thr-Lys-Arg ${ }^{75}$ ) [5]. The structure of furin includes a signal peptide, a propeptide, a subtilisin-like catalytic domain, a Homo B domain, a Cys-rich domain, a transmembrane domain and a cytoplasmic domain. Furin is capable of processing a wide variety of precursors including growth factors, hormones, neuropeptides, 
transcription factors, plasma proteins, receptors, viral envelope proteins, matrix metalloproteinases and bacterial exotoxins $[1,6]$. Many substrates of furin have important physiological functions and possess great clinical values. It is attractive for the pharmaceutical industry to produce these substrates via recombinant protein expression systems.

Pichia pastoris, reclassified as Komagataella phaffi [7], has become a successful host organism for production of recombinant proteins due to the benefits of high cell density cultivation, the simplicity of genetic manipulation, growth on inexpensive media, efficient secretory capabilities with a low level of endogenous protein secretion, the strong and tightly inducible $A O X 1$ promoter and the ability of post-translational modifications to proteins $[8,9]$. Currently, over 1000 proteins have been produced in P. pastoris [10]. In our previous studies, we heterologously expressed two furin substrates using the DNA sequences encoding the mature peptides in P. pastoris. But the recombinant proteins couldn't form the proper native structures $[11,12]$. Several studies have found that the propeptides in some precursors are important for the production of active mature peptides. The propeptides can act as intramolecular chaperones to assist the proper folding of mature peptides such as activin A and transforming growth factor-beta 1 (TGF- $\beta 1$ ) [13], bone morphogenetic protein-4 (BMP-4) [14] and cathepsin $\mathrm{L}$ [15], or stabilize the mature peptides to increase the their transport from ER to Golgi $[16,17]$. Therefore, coexpression of propeptide and mature peptide sequence may provide a strategy for efficient production of biologically active proteins processed by furin in P. pastoris. However, it is not clear whether the furin cleavage sites between the propeptide and mature peptide can be properly processed in $P$. pastoris, bringing uncertainty for proper expression of the DNA sequences of furin substrates containing the propeptides and mature peptides.

In this study, we used reporter proteins containing furin cleavage sites between the propeptide and mature peptide to systematically evaluate the ability of $P$. pastoris GS115 on processing furin cleavage sites. Our study will shed light on how to improve the efficiencies of producing biological active furin substrates in P. pastoris.

\section{Results and discussion}

\section{Furin cleavage sites can be cleaved in Pichia pastoris}

In our previous study, we examined all reported precursors processed by furin and found there were three main cleavage sites between propeptides and mature peptides: Arg-Xaa-Lys-Arg (41\%), Arg-Xaa-Arg-Arg (31.5\%) and Arg-Xaa-Xaa-Arg (11\%) (where Xaa is any amino acid) [18]. In order to determine if mammalian furin cleavage sites can be cleaved in $P$. pastoris, three reporter vectors containing different furin cleavage sites (Arg-SerLys-Arg, Arg-Ser-Arg-Arg or Arg-Ser-Ile-Arg) between $\alpha$-factor propeptide and GFP were constructed (Fig. 1a). The reporter vectors were introduced into $P$. pastoris GS115 by electroporation and the secreted proteins were subject to western blotting detection. A band indicating the cleaved GFP (about $27 \mathrm{kDa}$ ) can only be detected if furin cleavage sites could be cleaved in $P$. pastoris. The values of cleaved GFP band intensities divided by the total intensities of cleaved GFP band plus $\alpha$-factor-GFP fusion protein band were regarded as the cleavage efficiency. As shown in Fig. 1b, GFP proteins in the supernatants were detected at expected molecular weight in all three recombinant strains, indicating furin cleavage sites could be cleaved to release the GFP. Considering the cleavage efficiency is unlikely to reach $100 \%$, there should be some fusion proteins containing both $\alpha$-factor and GFP. However, there were no bands detected at the expected $36 \mathrm{kDa}$, instead some smear bands at higher molecular weight were observed (Fig. 1b). We suspected that the smear bands were $\mathrm{N}$-glycosylated fusion proteins of $\alpha$-factor and GFP because there are three potential $N$-glycosylation motifs in $\alpha$-factor peptide sequence. The culture supernatants were then treated with Endo $\mathrm{Hf}$, which cleaves oligosaccharides from $\mathrm{N}$-linked glycoproteins. The fusion proteins of $\alpha$-factor and GFP were detected at $36 \mathrm{kDa}$ after treatment (Fig. 1c). These results showed that all three furin cleavage sites can be partially cleaved in P. pastoris. The cleavage efficiency of Arg-SerLys-Arg motif was the highest among the three furin cleavage motifs (Fig. 1d).

Arg residues at position $\mathrm{P} 1$ and $\mathrm{P} 4$ in the cleavage site are essential for cleavage by furin in mammals $[19,20]$. In order to evaluate the site preference of the proteases in $P$. pastoris, reporter vectors containing mutation of Arg at P1 or P4 were constructed (Fig. 2a). When Arg residue at P1 or P4 in Arg-Ser-Ile-Arg motif was mutated into Ile, the cleavage was completely lost in P. pastoris (Fig. 2b, e). Mutation of Arg into Ile at P1 or P4 in both Arg-Ser-LysArg and Arg-Ser-Arg-Arg motifs significantly decreased the cleavage (Fig. 2c, d, f, g). Compared with Arg-Ser-IleArg, Arg-Ser-Lys-Arg and Arg-Ser-Arg-Arg motifs contain one more basic amino acid. After mutation of Arg at position P1 or P4 into Ile, there were still two basic amino acids in Arg-Ser-Lys-Arg and Arg-Ser-Arg-Arg motifs left, suggesting two basic amino acids are sufficient furin cleavage sites processing in P. pastoris. Moreover, the two basic amino acids do not have to be paired and can be separated by one or two other amino acids. The cleavage efficiencies of Arg-Ser-Ile-Arg motif (29\%) and Arg-SerArg-Arg motif (29.7\%), were similar, which were about two times lower than that of the Arg-Ser-Lys-Arg motif (49.3\%). Suggesting it is more favorable for furin cleavage 

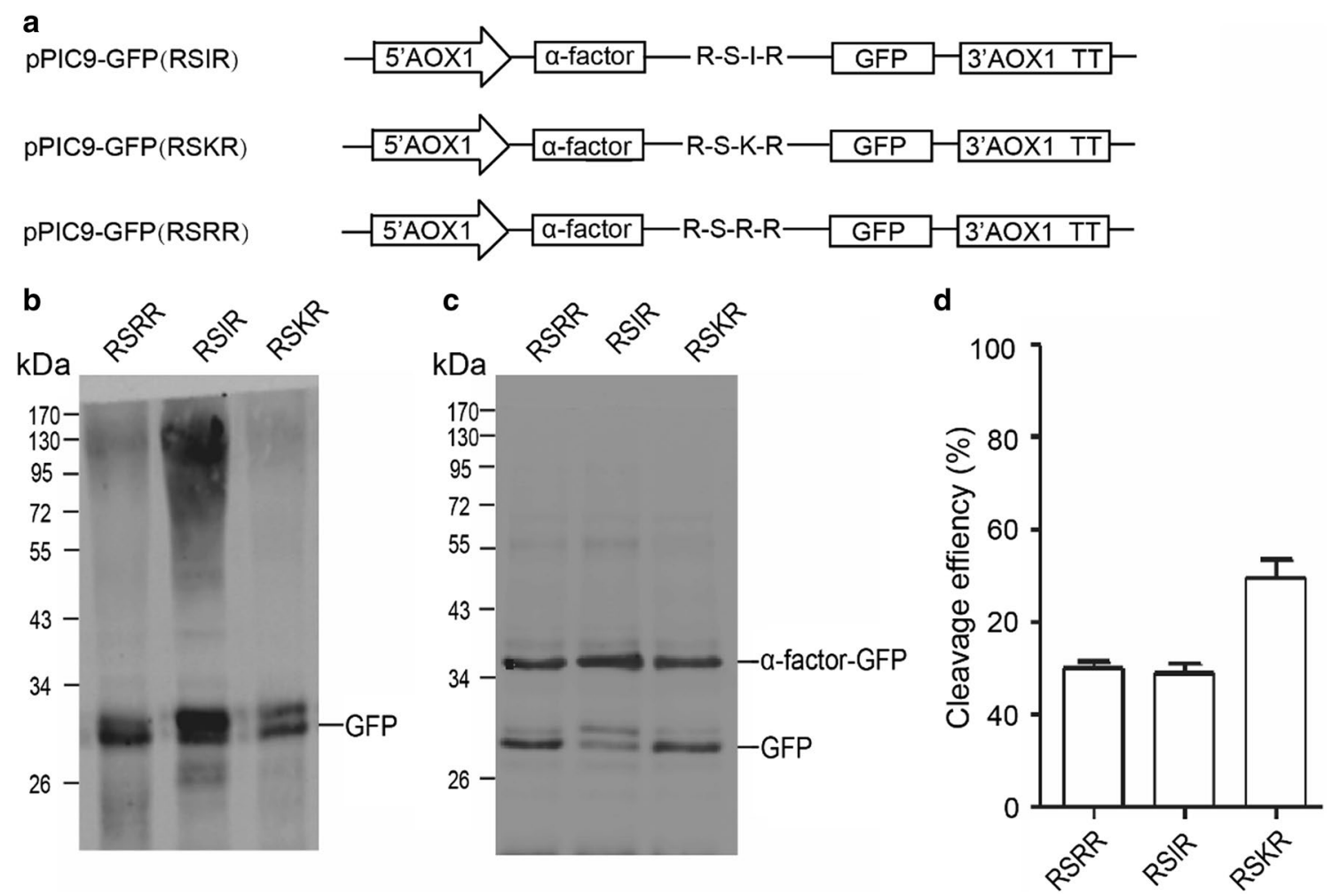

Fig. 1 Cleavage of furin cleavage site in Pichia pastoris. a Schematic diagram of expression vectors using in this study. b Western blot of expressed products using anti-GFP antibody, the culture supernatants didn't be treated with Endo Hf endoglycosidase which cleaves the chitobiose core of high mannose from N-linked glycoprotein. $\mathbf{c}$ Western blot of expressed products using anti-GFP antibody, the culture supernatants were treated with Endo $\mathrm{Hf}$ to show a-factor-GFP fusion protein after high mannose chains were removed from a-factor peptide. $\mathbf{d}$ Quantitative analysis of the western blot results, compared GFP with GFP and a-factor-GFP

site processing in $P$ pastoris if the P2 position is Lysine. The Arg-Xaa-Xaa-Arg motif is also cleaved by furin in mammal, but the cleavage efficiency is tenfold lower than that of Arg-Xaa-(Lys/Arg)-Arg motif [21-23]. The difference in the cleavage efficiency towards Arg-Xaa-Xaa-Arg motif between $P$. pastoris and mammal suggested the proteases in $P$. pastoris is more flexible than furin.

At P1 position, both furin and Saccharomyces cerevisiae Kex2 exhibit extremely stringent specificity for Arg. Studies on furin substrate specificity have also shown that replacement of P1 Arg results in undetectable cleavage [24]. Substitution with similar positive charge amino acid, such as Lys or ornithine, at P1 position in Saccharomyces cerevisiae Kex2 peptidyl-MCA substrates also resulted in an approximately 100 -fold drop in $\mathrm{k}_{\text {cat }} / \mathrm{K}_{\mathrm{M}}$ relative to the canonical substrate sequence [25]. The cleavage efficiencies of all three furin processing sites were also significantly decreased when Arg residue at position P1 was mutated into Ile. For Arg-Ser-Ile-Arg motif, the cleavage was completely abolished. For Arg-Ser-ArgArg and Arg-Ser-Lys-Arg motifs, the cleavage efficiencies were greatly decreased. Our results showed that Arg residue at P1 is essential for cleavage in P. pastoris. For Saccharomyces cerevisiae Kex2, most of its selectivity towards substrates arises through interactions at position P1 and P2 $[25,26]$. In P. pastoris, the amino acid at P2 seems to be less important if there is Arg at P4, suggesting that the selectivity of protease to substrates in $P$. pastoris is more like furin which generates most of its selectivity through interactions of Arg at P1 and P4.

\section{Kex2, but not YSP1, is responsible for processing the furin cleavage site in Pichia pastoris}

Our results indicated that furin cleavage sites can be processed in $P$. pastoris, but the exact proteases responsible for this processing are unclear. Genome sequencing of $P$. pastoris GS115 revealed the presence of a family of glycosylphosphatidylinositol (GPI)-linked aspartyl proteases named yapsins [27]. YPS1 (yapsin 1, previously named as Yap3; EC 3.4.23.41), a member of yapsins, was shown to cleave its substrates at mono- and paired basic amino acid residues $[28,29]$. Since there are mono- or paired basic amino acid residues in the three furin cleavage motifs, we first speculated that YSP1 is involved in the proteolytic 


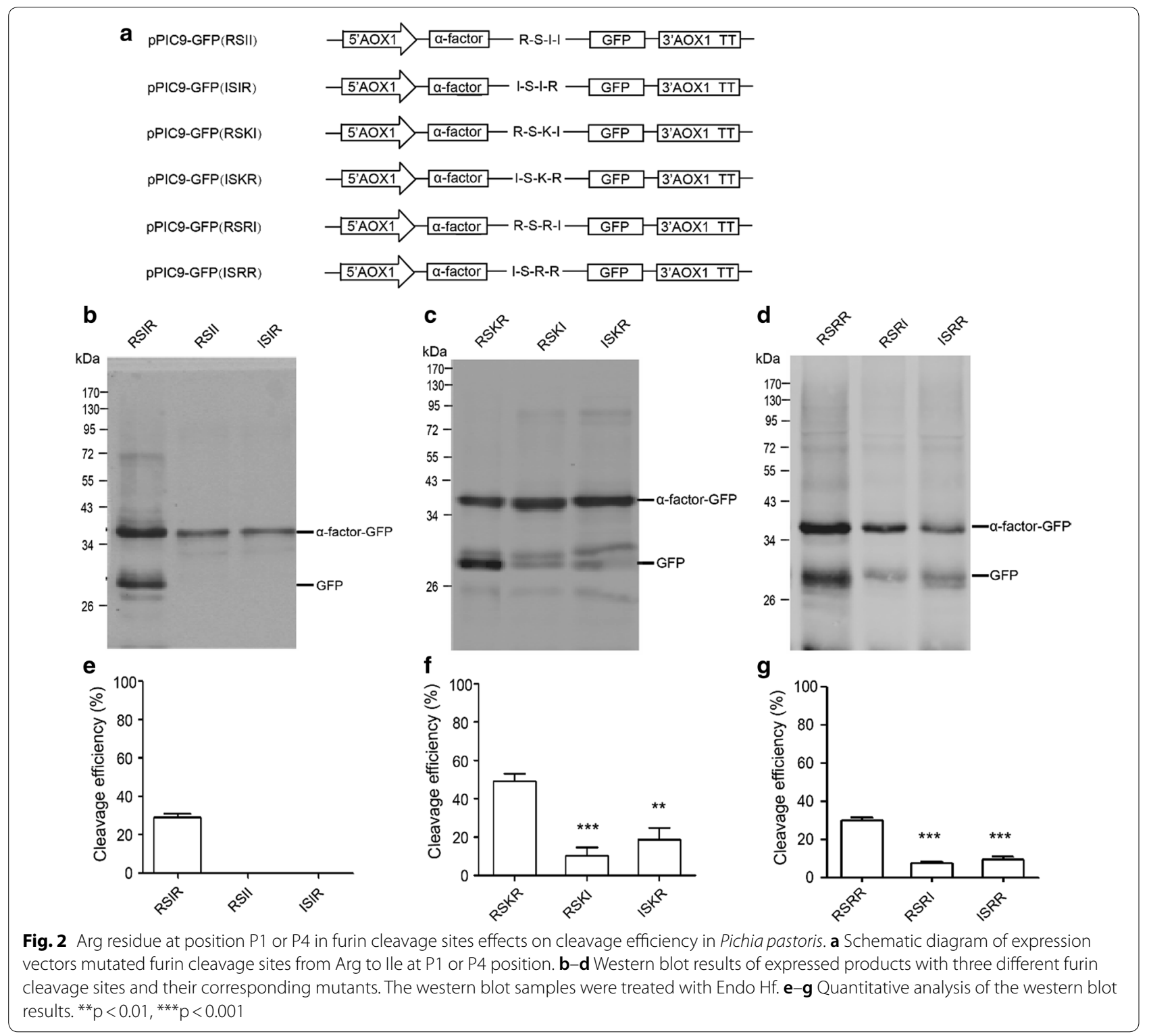

cleavage of furin cleavage sites. We constructed the disruption plasmid pYPS1 $\triangle$ (Fig. 3a), and YPS1 gene was disrupted by insertion of the zeocin-selectable plasmid pYPS1 $\triangle$ (Fig. 3b). Correct disruption of YPS1 gene was confirmed by PCR using two pairs of specific primers (Fig. 3c). The cleavage efficiencies in three furin cleavage sites were similar between wild type and the disruptants (Fig. 3d-f), indicating that YPS1 is not involved in the processing of furin cleavage sites in P. pastoris.

In addition to YPS1, we also investigated the effect of Kex2 protease on furin cleavage site processing. Kex2 is involved in processing $\alpha$-mating pheromone and killer toxin precursors by limited proteolysis at the carboxyl side of the paired basic amino acid residues [4, 30].
Biochemical analysis has also shown that Kex2 exhibits high activity toward substrates with Lys-Arg or Arg-Arg cleavage motifs [25, 31]. The Kex2 gene was disrupted by homologous recombination using a similar strategy for disrupting YPS1 gene (Fig. 4a-c). All of three furin cleavage sites were not cleaved in Kex2 disrupted strains (Fig. 4d, e), indicating Kex2 protease is responsible for the processing of furin cleavage sites in P. pastoris.

\section{Heterologous expression of furin or overexpression of Kex2 significantly increased cleavage efficiency of furin cleavage sites in Pichia pastoris}

Although endogenous Kex 2 in $P$. pastoris can process furin cleavage sites, the efficiency is not high. If the 
a
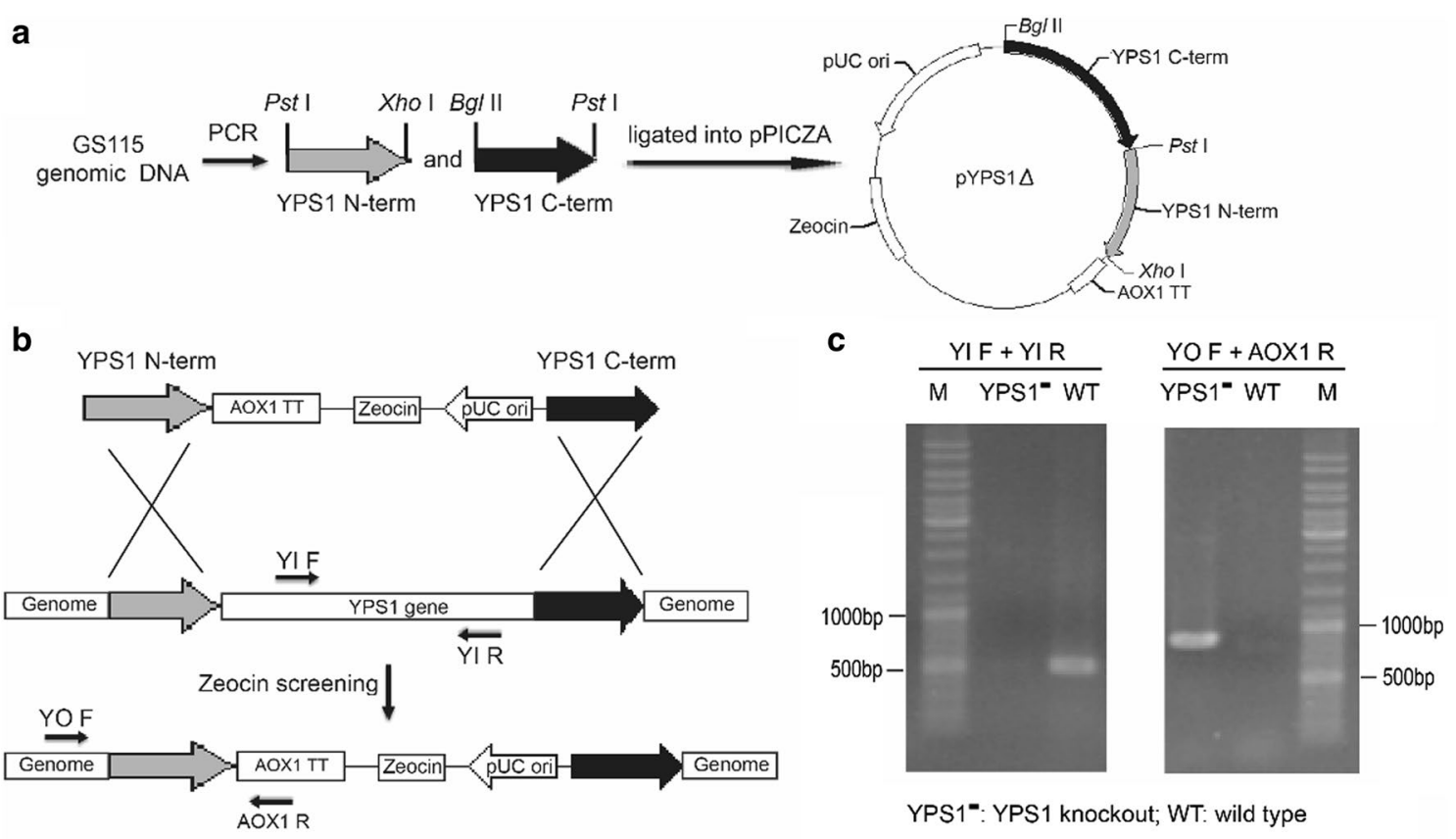

YPS1": YPS1 knockout; WT: wild type
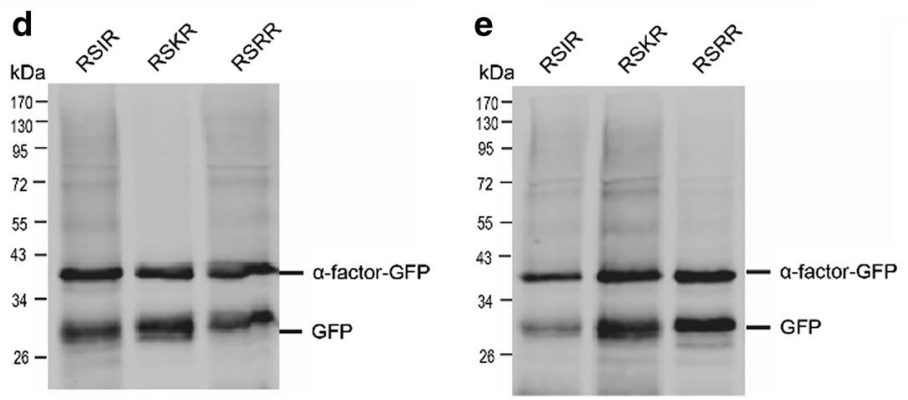

f

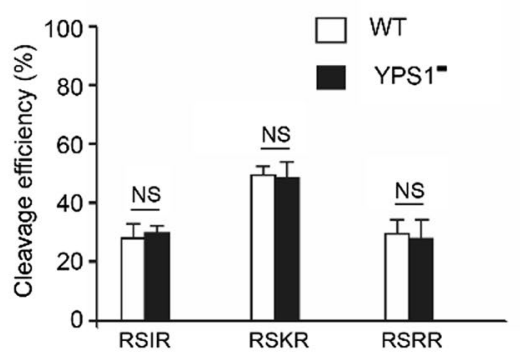

Fig. 3 YPS1 doesn't involve in processing the furin cleavage site in Pichia pastoris. a Schematic diagram for constructing a disruption vector with left homology arm and right homology arm of YPS1 gene. b Schematic diagram for knockout of YPS1 gene by homologous recombination. Zeocin can be used for selection of YPS1 knockout strains. Two pairs primers, as shown in figure, were designed to confirm the disrupted strains. c Genomic PCR of YSP1 disrupted and wild type strains using two pairs primers (YI F, YI R and YO F, AOX1 R). A band can be amplified by $Y I F$ and $Y I R$ primers only if YPS1 gene doesn't be deleted, and a band can be amplified by YO F and AOX1 primers if YPS1 gene is successfully removed by the disruption construct. $\mathbf{d}$ Western blot results of wild type strains. The western blot samples were treated with Endo Hf. e Western blot results of YPS1 disruption strains. The western blot samples were treated with Endo Hf. $\mathbf{f}$ Quantitative analysis of the western blot results. NS not statistically significant

DNA sequence of furin substrates were expressed in $P$. pastoris, the production of the mature peptides will be limited due to low cleavage efficacy. It will be useful to generate an engineered $P$. pastoris strain that is capable of efficient processing furin cleavage sites. Based on our above results, furin or Kex2 gene were integrated into P. pastoris genome. Cleavage efficiencies in three furin cleavage sites were evaluated in GS115-furin and GS115-Kex2 strains. As expected, the cleavage efficiencies in all three furin cleavage sites were significantly increased in both GS115-furin and GS115-Kex2 strains (Figs. 5 and 6), suggesting these two engineered strains can be applied for efficient production of furin substrates.
Recently, studies on ligase derived from fungus showed that co-expression of propeptide and mature peptide in $P$. pastoris helped mature peptide form the proper protein conformation [32,33]. A large number of propeptide of precursor proteins have been identified as intramolecular chaperones (IMCs) and assist the proper folding of mature peptides. Propeptides of precursors processed by furin were also found to be critical in correct protein folding of mature peptides [13, 14]. Further researches are needed to verify whether the expression of the furin substrate DNA sequence consisted of both the propeptide and mature peptide in $P$. pastoris help enhance to activity of the mature peptide. If this strategy proves to produce more active furin substates in comparison with 


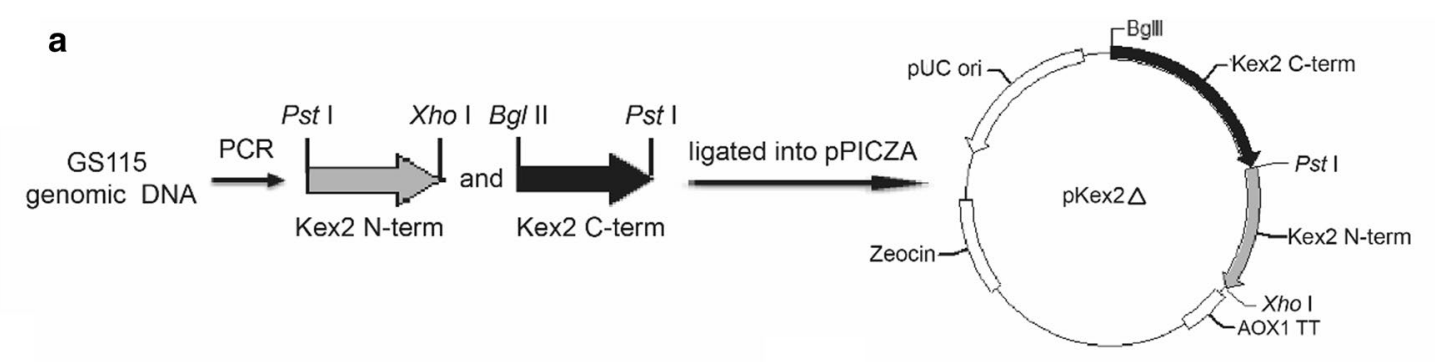

b
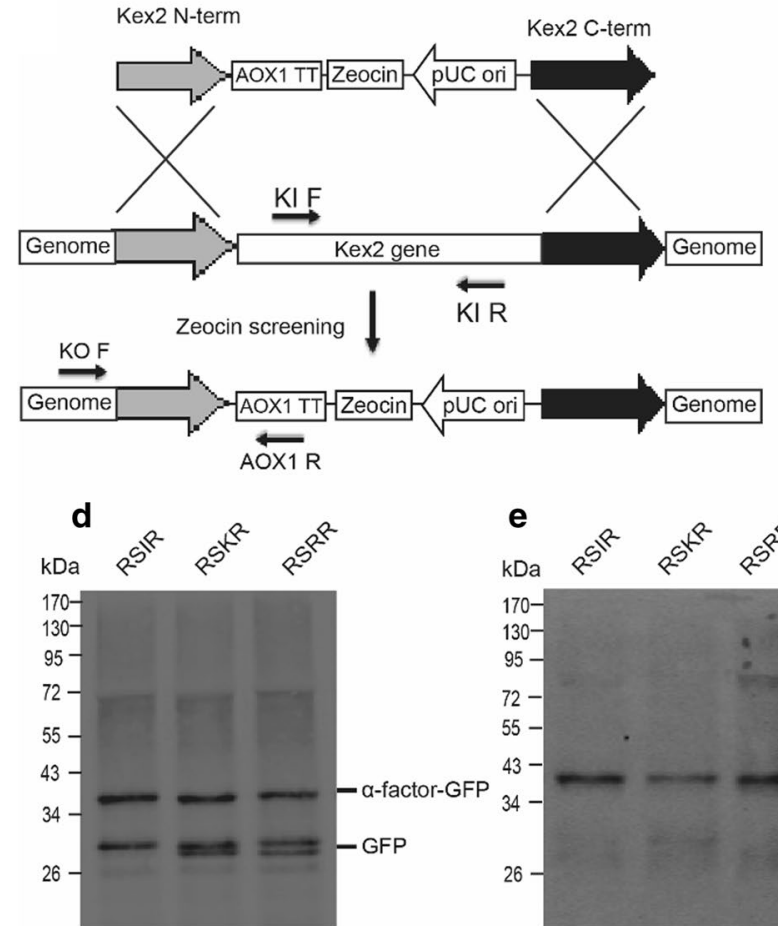

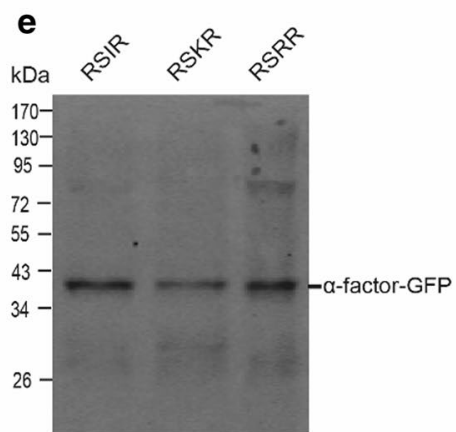

C

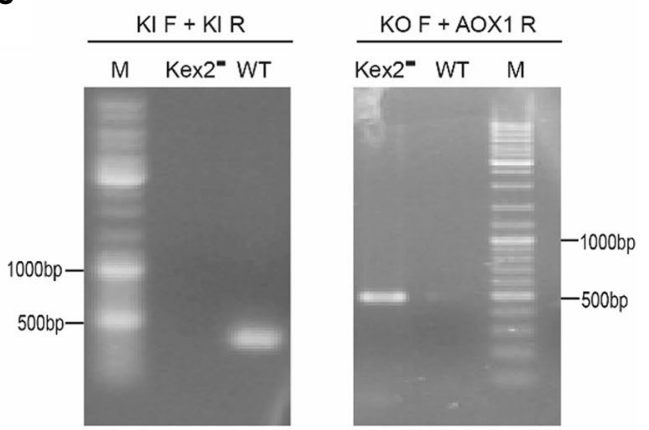

Kex2": Kex2 knockout; WT: wild type

f

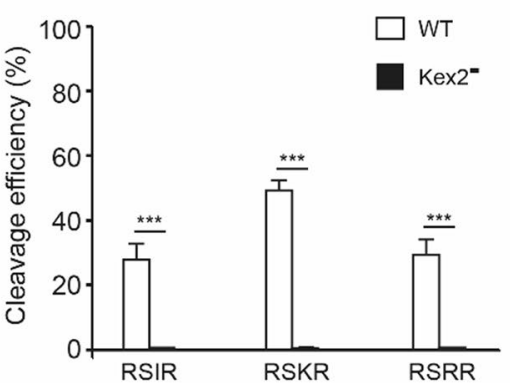

Fig. 4 Kex2 involved in processing the furin cleavage site in Pichia pastoris. a Schematic diagram for constructing a targeting vector with left homology arm and right homology arm of Kex2 gene. $\mathbf{b}$ Schematic diagram for knockout of Kex2 gene by homologous recombination. Zeocin can be used for selection of Kex2 disruption strains. Two pairs primers, as shown in figure, were designed to confirm the disruption strains. $\mathbf{c}$ Genomic $P C R$ of Kex2 disruption and wild type strains using two pairs primers ( $Y I F, Y I R$ and $Y O F, A O X 1 R$ ). A band can be amplified by $Y I F$ and $Y I R$ primers only if Kex2 gene doesn't be deleted, and a band can be amplified by YO F and AOX1 primers if Kex2 gene is successfully removed by the targeting construct. $\mathbf{d}$ Western blot results of wild type strains. The western blot samples were treated with Endo $\mathrm{Hf}$. e Western blot results of Kex2 disruption strains. The western blot samples were treated with Endo Hf. $\mathbf{f}$ Quantitative analysis of the western blot results. ${ }^{* *} \mathrm{p}<0.001$

direct expression of the mature peptide DNA sequence, our GS115-furin and GS115-Kex2 strains may be good choices for expressing precursors processed by furin in $P$. pastoris.

\section{Conclusions}

In this study we showed that Kex2, but not YPS1, in $P$. pastoris is capable of processing furin cleavage sites and the Arg residue at position P1 or P4 of furin cleavage sites significantly affect the cleavage efficiency. Overexpression of both furin or Kex2 in P. pastoris effectively increased the processing efficiency of furin cleavage sites, providing an attractive direction for efficiently express bioactive furin substrates in P. pastoris.

\section{Methods \\ Enzymes and reagents}

Restriction enzymes, T4 DNA ligase, Taq DNA polymerase, polymerase chain reaction (PCR) reagent, Prime STAR polymerase, and PCR reagent were obtained from Takara Bio Inc. The Endo Hf was purchased from New England Biolabs, Inc. SDS-PAGE Protein Marker was supplied by Thermo Fisher Scientific. Primers were all synthesized at Sangon Biotech (China). PCR Purification 

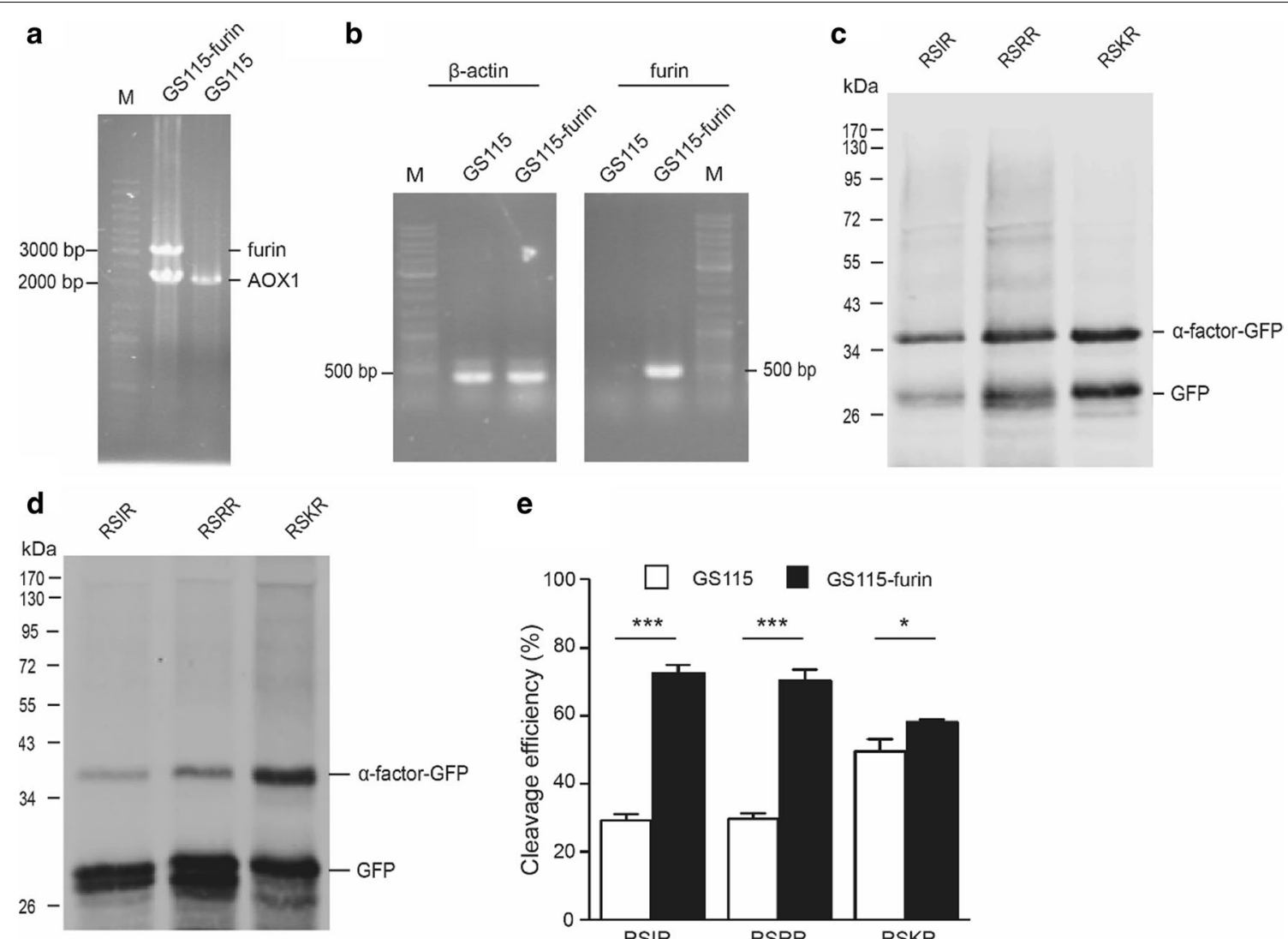

e

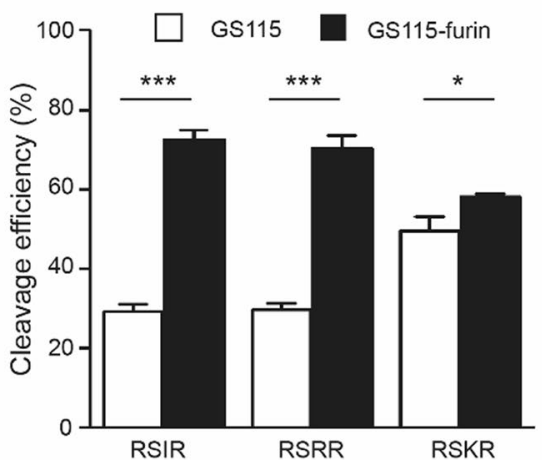

Fig. 5 Heterologous expression of furin increased cleavage efficiency of furin cleavage sites in Pichia pastoris. a Genomic PCR for analyzing integration of furin gene into GS115 genome using AOX1 general primers. b Expression analysis of integrated furin gene in Pichia pastoris GS115 by RT-PCR with $\beta$-actin and furin specific primers. $\mathbf{c}$ Western blot results of GS115 strains. The western blot samples were treated with Endo Hf. $\mathbf{d}$ Western blot results of GS115-furin strains. The western blot samples were treated with Endo Hf. e Quantitative western blot analysis of cleavage efficiency of three furin cleavage sites. ${ }^{*} p<0.05$, ${ }^{* *} p<0.001$

Kit was from Promega Corporation. Plasmid Mini Kit and Point mutation kit were from TransGen Biotech (China). All other chemicals used were acquired from Sigma Aldrich Chemicals P Ltd. and Sinopharm Chemical Reagent (China).

\section{Strains and plasmids}

Escherichia coli Top10F' was used as the cloning host. P. pastoris GS115(his $4^{-}$) was used as the expression host. Plasmid pPIC9 and pGAPZB were used as gene expression vectors. All of them were purchased from Thermo Fisher Scientific (Invitrogen). The recombinant plasmid pPIC9-GFP with different furin cleavage sites, YPS1 disruption vector pYPS1 $\Delta$, Kex2 disruption vector pKex $2 \Delta$, pGAPZB-Kex2 and pGAPZB-furin were constructed and stored in our lab.

\section{Preparation of DNA constructs}

All primer sequences using in this study were listed in Table 1. To construct plasmid pPIC9-GFP(RSIR),
GFP was amplified from pEGFP-N1 using a pair of primer, upstream primer with $\mathrm{XhoI}$ site and Arg-Ser-Ile$\operatorname{Arg}($ RSIR) motif sequence, and downstream primer with EcoRI site. The amplified fragment was cloned into pPIC9 vector at $X h o \mathrm{I}$ and EcoRI sites. The pPIC9-GFP(RSII), pPIC9-GFP(ISIR), pPIC9-GFP(RSRR) and pPIC9GFP(RSKR) were constructed through mutating RSIR motif of pPIC9-GFP(RSIR) plasmid using site-directed mutagenesis method, directly. pPIC9-GFP(RSRI) and pPIC9-GFP(ISRR) were generated using pPIC9GFP(RSRR) as template by site-directed PCR mutagenesis. Both of pPIC9-GFP(RSKI) and pPIC9-GFP(ISKR) were generated using pPIC9-GFP(RSKR) as template.

To construct YPS1 and Kex2 gene disruption vectors, The P. pastoris GS115 genome was extracted and used as template to amplify the left homology arm and right homology arm with two pairs of specific primers to YPS1 or Kex2 gene. The left arm fragment contains PstI site at $5^{\prime}$ end and $\mathrm{XhoI}$ at $3^{\prime}$ end, and the right arm fragment contains BglII site at $5^{\prime}$ end and PstI at 

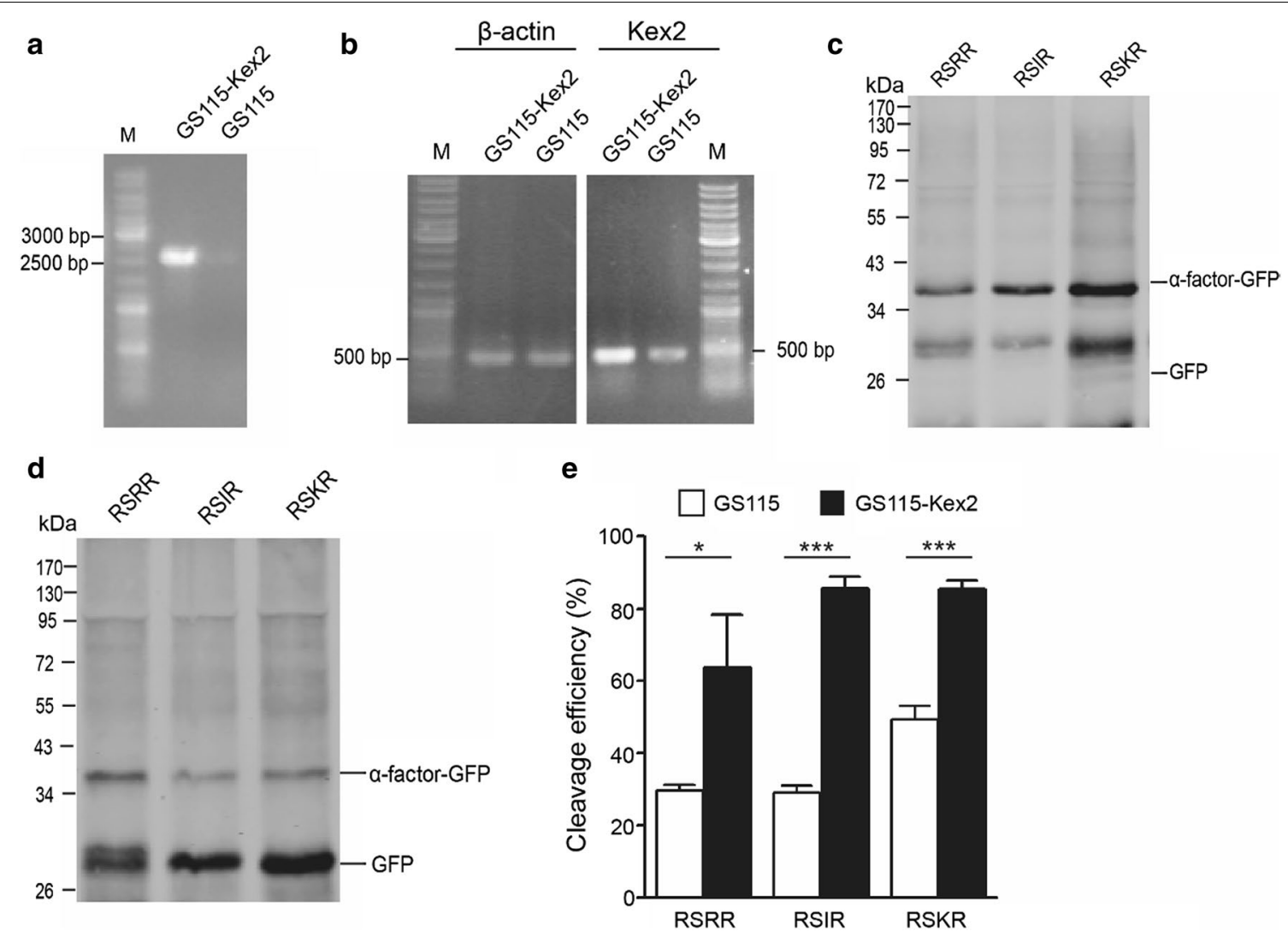

Fig. 6 Overexpression of Kex2 increased cleavage efficiency of furin cleavage sites in Pichia pastoris. a Genomic PCR for analyzing integration of Kex2 gene into GS115 genome using 5' GAP general upstream and Kex2 specific downstream primers. b Kex2 overexpression analysis in GS115-Kex2 strains by RT-PCR. Expression of $\beta$-actin was used as control. c Western blot results of GS115 strains. The western blot samples were treated with Endo Hf. $\mathbf{d}$ Western blot results of GS115-Kex2 strains. The western blot samples were treated with Endo Hf. e Quantitative western blot analysis of cleavage efficiency of three furin cleavage sites in GS115 and GS115-Kex2 strains. ${ }^{*} \mathrm{p}<0.05,{ }^{* * *} \mathrm{p}<0.001$

$3^{\prime}$ end. pPICZ $\alpha$ A was used as the backbone to generate the disruption vectors. Both of pPICZ $\alpha$ A and the left arm fragment were digested with the PstI and XhoI restriction endonucleases and ligated to produce an intermediate plasmid, The intermediate plasmid then was digested with the PstI and BglII restriction endonucleases. The right arm fragment digested with the same restriction endonucleases was inserted into the intermediate plasmid at the PstI and BglII sites to produce the disruption vector.

In order to overexpress Kex 2 protease in P. pastoris GS115, Kex2 gene was amplified from P. pastoris GS115 genome by PCR and cloned into pGAPZ B expression vector at EcoRI and XhoI sites. To heterologous expression of furin proprotein convertase in GS115, furin cDNA was amplified by PCR using pGEM-furin plasmid (a gift kindly provided by professor Gary Thomas from Department of Cell and Developmental Biology, Oregon Health and Science University, School of
Medicine, Portland, USA) as the template, and cloned into pGAPZ B expression vector at EcoRI and XhoI sites.

\section{Electroporation}

The recombinant plasmids pPIC9-GFP(RSIR), pPIC9GFP(RSRR), pPIC9-GFP(RSKR), pPIC9-GFP(ISIR), pPIC9-GFP(RSII), pPIC9-GFP(ISRR), pPIC9-GFP(RSRI), pPIC9-GFP(ISKR) and pPIC9-GFP(RSKI) were linearized with SalI restriction endonuclease. pKex $2 \Delta$, pYPS1 $\Delta$ were linearized with Pst I restriction endonuclease. pGAPZ B-Kex2 and pGAPZ B-furin were linearized with AvrII restriction endonuclease. $10 \mu \mathrm{g}$ linearized plasmid DNA was transformed into P. pastoris GS115 competent cells by electroporation $(1.5 \mathrm{kV}, 25 \mu \mathrm{F}, 200 \Omega)$ using a Bio-Rad Gene Pulser (Hercules, USA). The transformed cells were screened on MD $(0.34 \% \mathrm{YNB}, 4 \times 10-5 \%$ biotin, $1 \%$ dextrose, and $1.5 \%$ agar) or YPD (1\% yeast extract, $2 \%$ peptone, $2 \%$ dextrose and $1.5 \%$ agar) in the presence of $25 \mu \mathrm{g} / \mathrm{ml}$ Zeocin (Invitrogen) plates. 
Table 1 Primers using in this study

\begin{tabular}{|c|c|c|c|}
\hline Description & Primer name & Primer sequence $\left(5^{\prime}-3^{\prime}\right)$ & Restriction site \\
\hline \multicolumn{4}{|c|}{ Preparation for expression vectors } \\
\hline \multirow[t]{2}{*}{ pPIC9-GFP(RSIR) } & RSIR F & CCGCTCGAGAGATCTATCAGAGTGAGCAAGGGCGAG & Xhol \\
\hline & RSIR R & CCGGAATTCTTACTTGTACAGCTCGTC & EcoRl \\
\hline \multirow[t]{2}{*}{ pPIC9-GFP(RSII) } & RSII F & CTCTCGAGAGATCTATAATAGTGAGCAAGGG & \\
\hline & $\mathrm{RS} \| \mathrm{R}$ & СCCTTGCTCACTATTATAGATCTCTCGAGAG & \\
\hline \multirow[t]{2}{*}{ pPIC9-GFP(ISIR) } & ISIR F & CTCTCGAGATATCTATAAGAGTGAGCAAGGG & \\
\hline & ISIRR & CCCTTGCTCACTCTTATAGATATCTCGAGAG & \\
\hline \multirow[t]{2}{*}{ pPIC9-GFP(RSRR) } & RSRR F & CTCTCGAGAGATCTAGAAGAGTGAGCAAGGG & \\
\hline & RSRR R & CCCTTGCTCACTCTTCTAGATCTCTCGAGAG & \\
\hline \multirow[t]{2}{*}{ pPIC9-GFP(RSRI) } & RSRI F & CTCTCGAGAGATCTAGAATAGTGAGCAAGGG & \\
\hline & RSRI R & СCCTTGCTCACTATTCTAGATCTCTCGAGAG & \\
\hline \multirow[t]{2}{*}{ pPIC9-GFP(ISRR) } & ISRR F & CTCTCGAGATATCTAGAAGAGTGAGCAAGGG & \\
\hline & ISRR F & СCCTTGCTCACTCTTCTAGATATCTCGAGAG & \\
\hline \multirow[t]{2}{*}{ pPIC9-GFP(RSKR) } & RSKR F & CTCTCGAGAGATCTAAGAGAGTGAGCAAGGG & \\
\hline & RSKR R & CCCTTGCTCACTCTCTTAGATCTCTCGAGAG & \\
\hline \multirow[t]{2}{*}{ pPIC9-GFP(RSKI) } & RSKI F & CTCTCGAGAGATCTAAGATAGTGAGCAAGGG & \\
\hline & RSKI R & СCCTTGCTCACTCTCTTAGATATCTCGAGAG & \\
\hline \multirow[t]{2}{*}{ pPIC9-GFP(ISKR) } & ISKRF & CTCTCGAGATATCTAAGAGAGTGAGCAAGGG & \\
\hline & ISKRR & СCСTTGCTCACTATCTTAGATCTCTCGAGAG & \\
\hline \multirow[t]{2}{*}{ pGAPZ B-Kex2 } & Kex2 F & CCGGAATTCCGGATGTATTTGCCAGCACTT & EcoRl \\
\hline & Kex2R & CCGCTCGAGCGGTTACAATGCCGCACGTTT & Xhol \\
\hline \multirow[t]{2}{*}{ pGAPZ B-furin } & Furin $\mathrm{F}$ & CCGGAATTCCGGATGGAGCTGAGGCCCTGG & EcoRl \\
\hline & Furin $\mathrm{R}$ & CCGCTCGAGTTAGAGGGCGCTCTGGTCTT & Xhol \\
\hline \multicolumn{4}{|c|}{ Preparation for disruption vectors } \\
\hline \multirow[t]{4}{*}{ pYPS1 $\triangle$} & Left arm F & TGCACTGCAGTAGCCGTTCCCGCGTGAAGA & Pstl \\
\hline & Left arm R & CCGCTCGAGCGGACTAGCATATGTGGATTCTAG & Xhol \\
\hline & Right arm F & GGAAGATCTTCCTCCATCCTCTTTGGAGGTGTG & Bg/ll \\
\hline & Right arm R & TGCACTGCAGTGCACTATACTATACACACG & Pstl \\
\hline \multirow[t]{4}{*}{ pKex2 $\triangle$} & Left arm F & TGCACTGCAGTGCAATGTATTTGCCAGCAC & Pstl \\
\hline & Left arm R & CCGCTCGAGCGGTGGTGGATGAAGCCCTTTAAT & Xholl \\
\hline & Right arm F & GGAAGATCTTCCACGGATATGGCAAGATCGATG & $B g / l l$ \\
\hline & Right arm R & TGCACTGCAGTGCATTACAATGCCGCACGTTTGGG & Pastl \\
\hline \multicolumn{4}{|c|}{ Identification for disrupted strains } \\
\hline \multirow[t]{4}{*}{ GS115- $\triangle$ Yps 1} & YIF & CAGTATGACAATTTGCCAGC & \\
\hline & $\mathrm{YIR}$ & TCCCCCGGTGTAATATGTTG & \\
\hline & YOF & GGACTTCAGCGTTCTGAGGG & \\
\hline & AOX1R & GCAAATGGCATTCTGACATCC & \\
\hline \multirow[t]{4}{*}{ GS115- $\triangle$ Kex 2} & $\mathrm{KIF}$ & GGCTTGCTCTGCTGTGATGA & \\
\hline & $\mathrm{KI} R$ & AGATCTTTGGCCTCCTGGG & \\
\hline & $\mathrm{KOF}$ & CACCTGAAACTTAATACTCT & \\
\hline & AOX1R & GCAAATGGCATTCTGACATCC & \\
\hline \multicolumn{4}{|c|}{ Identification for GS115-furin and GS115-Kex2 strains } \\
\hline \multirow[t]{2}{*}{ GS115-furin } & AOX1 F & GACTGGTTCCAATTGACAAGC & \\
\hline & AOX1R & GCAAATGGCATTCTGACATCC & \\
\hline \multirow[t]{2}{*}{ GS115-Kex2 } & GAP F & GTCCCTATTTCAATCAATTGAA & \\
\hline & Kex2 R & GCCTCCTGGGTCAATTCATA & \\
\hline \multicolumn{4}{|l|}{ RT-PCR } \\
\hline$\beta$-actin & $\beta$-actin F & CTCCAATGAACCCAAAGTCCAAC & \\
\hline & $\beta$-actin R & GACAAAACGGCCTGAATAGAAAC & \\
\hline
\end{tabular}


Table 1 (continued)

\begin{tabular}{lll}
\hline Description & Primer name & Primer sequence $\mathbf{( 5}^{\prime} \mathbf{-} \mathbf{3}^{\prime} \mathbf{)}$ \\
\hline Furin & RT-furin $\mathrm{F}$ & GGCATTGTGGTCTCCATTCT \\
& RT-furin $\mathrm{R}$ & GCAGTTGCAGCTGTCATGTT \\
Kex2 & RT-Kex2 F & GGCATTGTGGTCTCCATTCT \\
& RT-Kex2 R & GCAGTTGCAGCTGTCATGTT \\
\hline
\end{tabular}

\section{Expression of protein in shaking flasks}

Colonies were picked into BMGY medium (1\% yeast extract, $2 \%$ peptone, $0.34 \% \mathrm{YNB}, 4 \times 10^{-5} \%$ biotin, $0.5 \%$ methanol, $100 \mathrm{mM}$ potassium phosphate, $\mathrm{pH} 6.0$,) and incubated in an orbital shaker at $220 \mathrm{rpm}$ for $24 \mathrm{~h}$ until the $\mathrm{OD}_{600 \mathrm{~nm}}$ reached 2-5. The supernatant was discarded by centrifugation at $1500 \mathrm{rcf}$ for $10 \mathrm{~min}$ at room temperature, and the cells were resuspended in $10 \mathrm{ml}$ BMMY medium (1\% yeast extract; $2 \%$ peptone; $100 \mathrm{mM}$ phosphate buffer, $\mathrm{pH} 6.0 ; 1.34 \%$ yeast nitrogen base, $4 \times 10^{-5} \%$ biotin; and $1 \%$ methanol) to a final $\mathrm{OD}_{600 \mathrm{~nm}}=1$ in $250 \mathrm{ml}$ shaking flasks. Methanol was added to induce expression of GFP every $24 \mathrm{~h}$. After shaking in flasks for $72 \mathrm{~h}$, the supernatant was harvested.

\section{Deglycosylation by Endo Hf}

Due to the presence of glycosylation sites in the $\alpha$-factor peptide, the expressed $\alpha$-factor-GFP fusion protein was glycosylated. Endo Hf (New England Biolabs) was used to remove $N$-glycan of $\alpha$-factor-GFP fusion protein. Briefly, the culture supernatant was boiled for $10 \mathrm{~min}$ in denatured buffer containing 0.4 M DTT and 0.5\% SDS, and deglycosylation was then performed by treatment with Endo $\mathrm{Hf}$ at $37{ }^{\circ} \mathrm{C}$ for overnight according to the instructions of the manufacture. The buffer employed in these enzyme reactions was $50 \mathrm{mM}$ sodium citrate ( $\mathrm{pH}$ 5.5) for Endo Hf.

\section{Determination of the cleavage efficiency of furin cleavage sites in Pichia pastoris by western blotting}

Cleavage efficiency to different furin cleavage sites in $P$. pastoris was validated by western blotting. The proteins in culture supernatant were separated by SDS-PAGE (12\% gel), and then transferred onto a Protran nitrocellulose membrane (Signa Amersham) using a Mini TransBlot Cell (Bio-Rad). The membranes were blocked with $5 \%$ fat-free milk and incubated overnight at $4{ }^{\circ} \mathrm{C}$ with anti-GFP antibodies at 1:500 dilutions (Santa Cruz Biotechnology, Santa Cruz, CA, USA). IRDye $800 \mathrm{CW}$ goat anti-mouse immunoglobulin G (LI-COR Biosciences) was used as the secondary antibody at 1:1000 dilutions. Detection and quantification of proteins were performed using Odyssey imaging systems (LI-COR Biosciences). Cleavage efficiency was calculated by the following equation: cleavage efficiency $=$ sum of cleaved GFP band intensities/sum of cleaved GFP and $\alpha$-factor-GFP fusion protein band intensities. All the experiments were repeated at least three times independently.

\section{RT-PCR}

Total RNA of Kex2 or furin knockin GS115 strains was obtained using Yeast RNAiso Kit (Takara Bio Inc.) and cDNA was synthesized from 500 ng of the total RNA using Reverse Transcription System (Takara Bio Inc.). The primer sequences used in this work are shown in Table 1. The PCR mixture was first heated to $95{ }^{\circ} \mathrm{C}$ for $5 \mathrm{~min}$, then entering 30 cycles of $94{ }^{\circ} \mathrm{C}$ for $30 \mathrm{~s}, 55^{\circ} \mathrm{C}$ for $30 \mathrm{~s}$ and $72{ }^{\circ} \mathrm{C}$ for $30 \mathrm{~s}$. The PCR products were analyzed by $1.5 \%$ agarose gel electrophoresis with ethidium bromide staining.

\section{Authors' contributions}

YH and YLi conceived and designed the experiments. YLo performed the majority of the laboratory work. SL and TL mutated furin cleavage sites. JW and YZ constructed plasmids. YH wrote the manuscript. YLi revised the manuscript. All authors read and approved the final manuscript.

\section{Author details}

${ }^{1}$ Provincial University Key Laboratory of Cellular Stress Response and Metabolic Regulation, College of Life Sciences, Fujian Normal University, Fuzhou 350007, China. ${ }^{2}$ Key Laboratory of Optoelectronic Science and Technology for Medicine of Ministry of Education, Fujian Normal University, Fuzhou 350007, China.

\section{Acknowledgements \\ The authors would especially like to thank Prof. Gary Thomas for the kind gift} of pGEM-furin plasmid.

\section{Competing interests}

The authors declare that they have no competing interests.

Availability of data and materials

The data supporting the conclusions of this article are included with the article.

\section{Consent for publication \\ Not applicable.}

Ethics approval and consent to participate Not applicable.

Funding

This project is financed by the Natural Science Foundation of Fujian Province (2016J01146) and the Scientific Research Innovation Team Construction Program of Fujian Normal University (IRTL1702). 


\section{Publisher's Note}

Springer Nature remains neutral with regard to jurisdictional claims in published maps and institutional affiliations.

Received: 29 August 2018 Accepted: 31 October 2018

Published online: 08 November 2018

\section{References}

1. Nakayama K. Furin: a mammalian subtilisin/Kex2p-like endoprotease involved in processing of a wide variety of precursor proteins. Biochem J. 1997:327(Pt 3):625-35.

2. Seidah NG, Prat A. The biology and therapeutic targeting of the proprotein convertases. Nat Rev Drug Discov. 2012:11:367-83.

3. Fuller RS, Sterne RE, Thorner J. Enzymes required for yeast prohormone processing. Annu Rev Physiol. 1988:50:345-62.

4. Fuller RS, Brake AJ, Thorner J. Intracellular targeting and structural conservation of a prohormone-processing endoprotease. Science. 1989;246:482-6.

5. Anderson ED, VanSlyke JK, Thulin CD, Jean F, Thomas G. Activation of the furin endoprotease is a multiple-step process: requirements for acidification and internal propeptide cleavage. EMBO J. 1997;16:1508-18.

6. Jaaks $\mathrm{P}$, Bernasconi M. The proprotein convertase furin in tumour progression. Int J Cancer. 2017;141:654-63.

7. Kurtzman CP. Biotechnological strains of Komagataella (Pichia) pastoris are Komagataella phaffii as determined from multigene sequence analysis. J Ind Microbiol Biotechnol. 2009;36:1435-8.

8. Cereghino GP, Cereghino JL, llgen C, Cregg JM. Production of recombinant proteins in fermenter cultures of the yeast Pichia pastoris. Curr Opin Biotechnol. 2002;13:329-32.

9. Cregg JM, Cereghino JL, Shi J, Higgins DR. Recombinant protein expression in Pichia pastoris. Mol Biotechnol. 2000;16:23-52.

10. Theron CW, Berrios J, Delvigne F, Fickers P. Integrating metabolic modeling and population heterogeneity analysis into optimizing recombinant protein production by Komagataella (Pichia) pastoris. Appl Microbiol Biotechnol. 2018;102:63-80.

11. Huang Y, Zhen B, Lin Y, Cai Y, Lin Z, Deng C, Zhang Y. Expression of codon optimized human bone morphogenetic protein 4 in Pichia pastoris. Biotechnol Appl Biochem. 2014;61:175-83.

12. Chen L, Huang YD, Zhang YD. Expression and secretion of human bone morphogenetic protein-7 in Pichia pastoris. Sheng Wu Gong Cheng Xue Bao. 2006;22:907-13.

13. Gray AM, Mason AJ. Requirement for activin A and transforming growth factor-beta 1 pro-regions in homodimer assembly. Science. 1990;247:1328-30.

14. Degnin C, Jean F, Thomas G, Christian JL. Cleavages within the prodomain direct intracellular trafficking and degradation of mature bone morphogenetic protein-4. Mol Biol Cell. 2004;15:5012-20.

15. Smith SM, Gottesman MM. Activity and deletion analysis of recombinant human cathepsin L expressed in Escherichia coli. J Biol Chem. 1989:264:20487-95

16. Pepinsky B, Gong BJ, Gao Y, Lehmann A, Ferrant J, Amatucci J, Sun $Y$, Bush M, WalzT, Pederson N, et al. A prodomain fragment from the proteolytic activation of growth differentiation factor 11 remains associated with the mature growth factor and keeps it soluble. Biochemistry. 2017:56:4405-18.

17. Holst B, Bruun AW, KiellandBrandt MC, Winther JR. Competition between folding and glycosylation in the endoplasmic reticulum. EMBO J. 1996;15:3538-46.
18. Long YLY, Huang Y. Proprotein convertase furin and its processed preproprotein. Chin J Pharm Biotechnol. 2017;24:9.

19. Thomas $G$. Furin at the cutting edge: from protein traffic to embryogenesis and disease. Nat Rev Mol Cell Biol. 2002;3:753-66.

20. Takahashi S, Hatsuzawa K, Watanabe T, Murakami K, Nakayama K. Sequence requirements for endoproteolytic processing of precursor proteins by furin: transfection and in vitro experiments. J Biochem. 1994;116:47-52.

21. Klimpel KR, Molloy SS, Thomas G, Leppla SH. Anthrax toxin protective antigen is activated by a cell surface protease with the sequence specificity and catalytic properties of furin. Proc Natl Acad Sci USA. 1992;89:10277-81.

22. Molloy SS, Bresnahan PA, Leppla SH, Klimpel KR, Thomas G. Human furin is a calcium-dependent serine endoprotease that recognizes the sequence Arg-X-X-Arg and efficiently cleaves anthrax toxin protective antigen. J Biol Chem. 1992;267:16396-402.

23. Hipp MM, Shepherd D, Booth S, Waithe D, e Sousa CR, Cerundolo V. The processed amino-terminal fragment of human TLR7 acts as a chaperone to direct human TLR7 into endosomes. J Immunol. 2015;194:5417-25.

24. Rockwell NC, Krysan DJ, Komiyama T, Fuller RS. Precursor processing by kex2/furin proteases. Chem Rev. 2002;102:4525-48.

25. Rockwell NC, Wang GT, Krafft GA, Fuller RS. Internally consistent libraries of fluorogenic substrates demonstrate that Kex 2 protease specificity is generated by multiple mechanisms. Biochemistry. 1997;36:1912-7.

26. Rockwell NC, Fuller RS. Interplay between S1 and S4 subsites in Kex2 protease: Kex2 exhibits dual specificity for the P4 side chain. Biochemistry. 1998;37:3386-91.

27. De Schutter K, Lin YC, Tiels P, Van Hecke A, Glinka S, Weber-Lehmann J, Rouze P, Van de Peer Y, Callewaert N. Genome sequence of the recombinant protein production host Pichia pastoris. Nat Biotechnol. 2009:27:561-6.

28. Azaryan AV, Wong M, Friedman TC, Cawley NX, Estivariz FE, Chen HC, Loh YP. Purification and characterization of a paired basic residue-specific yeast aspartic protease encoded by the YAP3 gene. Similarity to the mammalian pro-opiomelanocortin-converting enzyme. J Biol Chem. 1993;268:11968-75.

29. Kang HA, Kim SJ, Choi ES, Rhee SK, Chung BH. Efficient production of intact human parathyroid hormone in a Saccharomyces cerevisiae mutant deficient in yeast aspartic protease 3 (YAP3). Appl Microbiol Biotechnol. 1998:50:187-92.

30. Julius D, Brake A, Blair L, Kunisawa R, Thorner J. Isolation of the putative structural gene for the lysine-arginine-cleaving endopeptidase required for processing of yeast prepro-alpha-factor. Cell. 1984;37:1075-89.

31. Brenner C, Fuller RS. Structural and enzymatic characterization of a purified prohormone-processing enzyme: secreted, soluble Kex2 protease. Proc Natl Acad Sci USA. 1992;89:922-6.

32. Cai H, Zhao M, Li Y, Mao J, Cai C, Feng F. Pentapeptide prosequence enhances expression and structure folding of recombinant thermomyces lanuginosus lipase in Pichia pastoris. Protein Pept Lett. 2017;24:676-81.

33. Wang Z, Lv P, Luo W, Yuan Z, He D. Expression in Pichia pastoris and characterization of Rhizomucor miehei lipases containing a new propeptide region. J Gen Appl Microbiol. 2016;62:25-30.

Ready to submit your research? Choose BMC and benefit from:

- fast, convenient online submission

- thorough peer review by experienced researchers in your field

- rapid publication on acceptance

- support for research data, including large and complex data types

- gold Open Access which fosters wider collaboration and increased citations

- maximum visibility for your research: over $100 \mathrm{M}$ website views per year

At BMC, research is always in progress.

Learn more biomedcentral.com/submissions 